\title{
Progress in Executive-Function Research
}

\section{From Tasks to Functions to Regions to Networks}

\author{
Adam R. Aron \\ University of California, San Diego
}

\begin{abstract}
It has long been observed that damage to the frontal cortex affects a person's ability to control thought, behavior, and emotion while sometimes leaving fundamental processes such as vision, hearing, and long-term memory intact. Such observations have led theoreticians to suppose that a set of executive control functions exists, at the top of the hierarchy of mental processes. To study these executive functions and their relation to the frontal cortex and its subregions, researchers have long employed several now-classic cognitive tests in patients with brain damage. Yet until recently it has proved difficult to reliably localize the putative executive functions to discrete regions. This article illustrates how recent progress in executive-functions research has been driven by the coupling of sophisticated neuroscience techniques with advances in experimental psychology. Taking examples from recent studies, it shows how experimental tasks may be decomposed into cognitive components that can be localized to discrete-but structurally connected-brain regions. What emerges is a new ontology for executive function in terms of which cognitive components exist and of how, and when, they are recruited during task performance.
\end{abstract}

KEYWORDS-executive functions; frontal cortex

Executive functions are higher-order cognitive functions that stand at the apogee of both evolutionary and mental development. They enable us to formulate goals and plans; remember these goals across time; choose and initiate actions to help us achieve these goals; and monitor and adjust our behavior, as necessary, until we complete or fail at them. The idea that such executive functions exist came from observing people with brain damage, typically damage to their frontal lobes. In some cases,

Address correspondence to Adam R. Aron, Department of Psychology, University of California, San Diego, 9500 Gilman Drive, La Jolla, CA 92093; e-mail: adamaron@ucsd.edu. including the famous case of Phineas Gage (the railroad worker whose frontal lobe was penetrated by a projectile), it was strikingly obvious that some "higher" cognitive power had been lost even when vision, audition, feeling, movement, speech, and long-term memory were intact.

The experimental study of executive functions began in earnest when several now-classic tasks were administered to patients with frontal-lobe damage. Such tasks included the Wisconsin Card Sort Test (WCST), the Stroop test, and tests of working memory and planning. The WCST requires subjects to override a tendency to sort cards according to a previously relevant category and instead to start sorting cards according to a new, to-be-discovered, correct category. In the Stroop task, subjects have to override the prepotent tendency to read a word in order to name the color in which the word is written (something that is more difficult because less habitual). Investigators showed repeatedly that damage to various regions of the frontal lobe produced behavioral impairment on such tests, particularly for conditions requiring overcoming prepotent response tendencies, but the results were often inconsistent. Some of the inconsistency was probably related to gross differences among tasks. If such gross differences exist then different tasks may tap underlying psychological components in different ways, and the relation to particular brain regions is difficult to establish. It was thus supposed that the outlook for such research could be improved if executive functions could be decomposed into constructs such as "initiating," "sustaining," "rule maintenance," "switching," "inhibiting," and "monitoring." More refined tests were developed in both human and nonhuman animal experiments to try to map such components onto particular sectors of the frontal cortex (reviewed by Robbins, 1996).

Nevertheless, 10 years ago an assessment of the state of executive-function research found much to complain about (Rabbitt, 1997). Although particular tasks had been broken down into supposed components, it was unclear if these hypothetical processes had real construct value at the psychological 
or neural levels. It seemed that brain damage might affect tasks designed to tap executive functions not because of effects on functionally localized modules but because executive-function tasks are just somehow "more demanding" than non-executivefunction tasks. Further, the pattern of data relating different executive functions to brain regions was still quite inconsistent.

In this article, I aim to show that the intervening decade has seen rapid progress in executive-function research that is driven by two major, and strongly interacting, trends. The first trend is the adoption of experimental-psychology paradigms that provide measures of the putative cognitive functions that are more precise than "classical" neuropsychological tests. The new tests' precision makes functional operationalization more reliable within and across subjects and more sensitive to the effects of brain damage and physiology. The second trend is the adoption of cutting-edge neuroscience techniques in imaging, neurophysiology, and brain stimulation. Together, these methods have revived the possibility that individual executive functions are rooted in particular neural systems. Yet the picture that emerges is a complex one: of separate brain regions connected by direct pathways for fast transmission of common information. To illustrate this approach, and to spell out the implications for experimental psychology, I offer several examples from studies of response control.

\section{STOPPING AN INCIPIENT RESPONSE}

Imagine you are about to press your foot down on your car's accelerator but, as you begin, a bicycle appears in front of your car. Clearly you need to countermand the motor command. Like shifting in the WCST, and like the ability to overcome Stroop interference, such stopping requires control over a prepotent response. However, stopping an already-initiated (incipient) response is different from controlling other kinds of prepotent responses in a substantial way: When studied in the lab, experimentally, it allows a specific examination of the way in which the controlling (stopping) process interacts with the initiated impulse (or "go process"). A dependent measure is derived (see below) for the actual speed of the control process itself. This speed-of-stopping measure has highly useful characteristics for brain research.

Measuring the speed of the stopping process became possible with experimental paradigms such as the stop-signal task (Logan \& Cowan, 1984). In this task, subjects are instructed to respond as fast as possible to imperative (go) stimuli and to do their best to inhibit the incipient response when a stop signal subsequently occurs. If the delay between imperative stimulus and the stop signal is short, subjects are likely to inhibit; if the delay is long, subjects are less likely to inhibit. By varying the delays and employing a mathematical model of go and stop processes, it is possible to estimate how quickly the subject can stop-the stopsignal reaction time (SSRT; Logan \& Cowan, 1984).
The ability to derive a good behavioral index of stopping has had strong implications for neuroscience research. It has facilitated many studies (too numerous to detail here) in different species and using different methods (see Aron et al., 2007 for review). Importantly, SSRT offers features uncommon among its sister variables in executive-function research: good withinsubject reliability, good reliability across groups matched on age, good translational application in rodent and nonhuman primate models, and good sensitivity for detecting effects of pharmacological manipulation and brain lesion.

My colleagues and I tried to find out which, if any, region of the prefrontal cortex is critical for stopping by examining SSRT in patients with brain damage. We found that the inferior frontal cortex (IFC) in the right hemisphere is critical for stopping (reviewed in Aron, 2007). In the initial study, damage to other sectors of either the right or left frontal cortex seemed unimportant, but recent work also points to an area at the top and middle of the brain, the presupplementary motor area (preSMA; Floden \& Stuss, 2006; Nachev, Wydell, O’Neill, Husain, \& Kennard, 2007); the relevance of this latter finding will be made clear later in this article.

Subsequent studies with functional magnetic resonance imaging (fMRI) have shown, moreover, that the degree of activation in the right IFC predicts the speed of stopping (Aron, Behrens, Smith, Frank, \& Poldrack, 2007; Aron \& Poldrack, 2006). This provides a neural correlate for a control process that can be examined with fMRI when other kinds of stopping are performed (eye, speech; see Xue, Aron, \& Poldrack, 2008) and when other kinds of control tasks are performed.

\section{A CIRCUIT FOR BEHAVIORAL AND NEURAL INHIBITION}

The foregoing shows that a particular sector of the right prefrontal cortex is important for stopping. Yet what is the significance of this for our psychological understanding? One way to answer this is to consider how the right IFC influences the incipient motor command. In the earlier example of driving, if you plan to press the accelerator with your foot and begin to activate your muscles, by what mechanism can the putative frontal command in the right IFC intercept the go command in the motor system? Does it do this by suppressing the motor command itself (and thereby inhibiting the agonist muscle), or does it activate an alternate representation (causing an antagonist muscle to block the action)?

Although it has long been supposed that a cardinal role for the frontal cortex is to inhibit and activate representations in posterior cortical or subcortical regions (Miller \& Cohen, 2001), there have been few demonstrations of underlying mechanisms. One way the stop process could intercept and block the go process is via the basal ganglia. Much research has shown that initiating a motor response engages the so-called "direct pathway" of the basal ganglia, in which the planning regions of the 
a

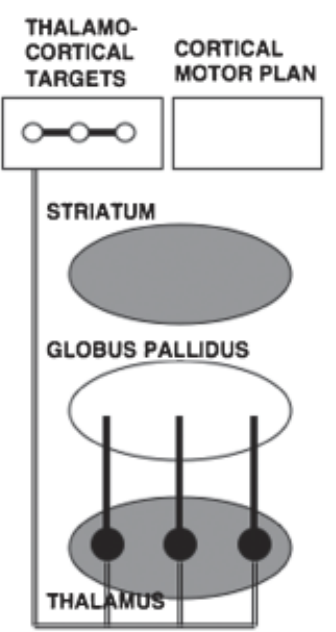

b

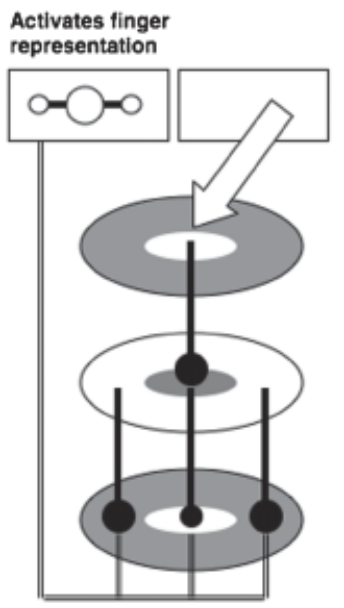

C

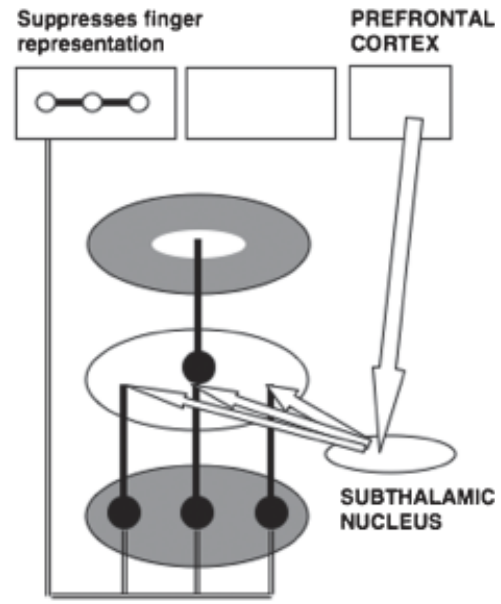

d

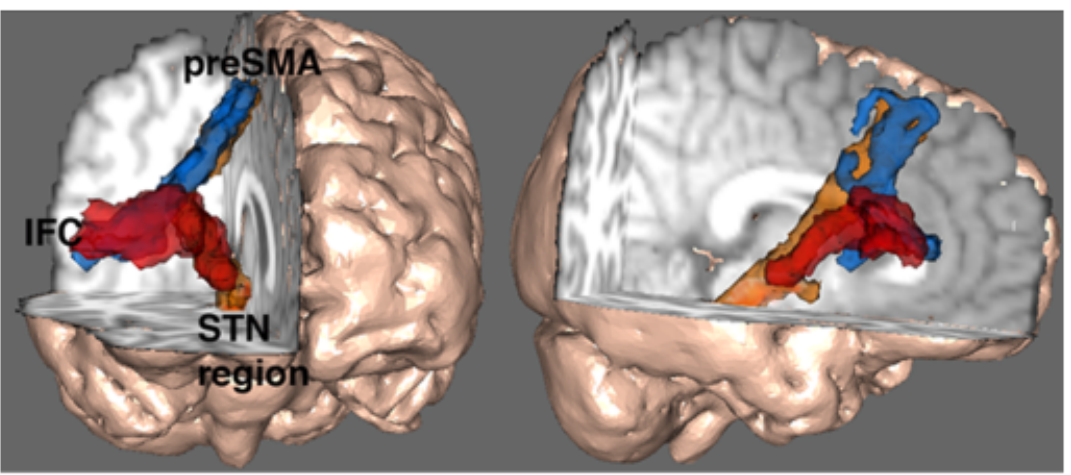

Fig. 1. Circuitry between the cortex and basal ganglia (a-c) and the brain's "stopping" or "braking" network discussed in the article (d). Filled heavy lines in the circuitry schematics are inhibitory connections; open arrows are excitatory connections. Information flows from the cortex through the basal ganglia and back to the cortex — in the diagram this means from top to bottom and back to top. At rest (a), striatal cells are quiescent, while pallidal neurons fire continually, thus suppressing thalamocortical output to the primary motor cortex (where finger and other representations reside). The motor command (large arrow in b) selectively excites a particular finger representation against the background of inhibition of irrelevant responses. One way in which the incipient response could be stopped (shown in c) is via the fast, hyperdirect, prefrontal-to-subthalamic route discussed in the article. A cutaway of the right hemisphere of the brain (viewed from the front and side; d) reveals white matter tracts or "cables" (colored) that connect three distant regions of the brain known to be important for controlling behavior. PreSMA = presupplementary motor area; IFC $=$ inferior frontal cortex; STN region $=$ midbrain region consistent with subthalamic nucleus. From "Triangulating a Cognitive Control Network Using Diffusion-Weighted Magnetic Resonance Imaging (MRI) and Functional MRI," by A.R. Aron, T.E. Behrens, S. Smith, M.J. Frank, \& R.A. Poldrack, 2007, Journal of Neuroscience, 27 (p. 3746). Copyright 2007, Society for Neuroscience. Reprinted with permission.

frontal cortex send activity to the putamen, which then projects to the globus pallidus, then to the thalamus, then to primary motor cortex, and onward to the muscles (Fig. la,b). We found activation in regions consistent with this direct pathway when subjects responded on go trials (Aron \& Poldrack, 2006). On stop trials, we found activation of a basal ganglia region in the vicinity of the subthalamic nucleus (STN), in addition to activation of the right IFC and preSMA (Aron \& Poldrack, 2006). This is striking because the STN has been conceived as a "stop button"-its activity levels are altered in Parkinson's disease, thus contributing to rigidity (and tremor). The STN projects to the globus pallidus, thus increasing inhibition of the thalamus and blocking basal ganglia output to the primary motor cortex (Fig. 1c).

How might the prefrontal stopping command get to the STN in the basal ganglia? We used a form of structural magnetic resonance imaging called diffusion weighted imaging to establish that these regions are directly connected via a white-matter tract (Aron et al., 2007; Fig. 1d). Thus, the same frontal region that is critical for stopping apparently sends axons into a region of the basal ganglia, the STN, that increases excitation of the globus pallidus and so could block the go command. Could this mechanism of control simply work by activating the antagonist 
representation? Probably not. A study that measured the activity level of the cortical representation of muscles during a stopsignal task showed that both the agonist muscle as well as an irrelevant muscle representation in the cortex were suppressed by the requirement to inhibit the incipient response (Coxon, Stinear, \& Byblow, 2006). This suggests that stopping is achieved via active inhibition of (potential) response tendencies rather than via activation of the antagonist muscle.

These data provide a mechanistic account of how the frontal cortex can interact with the motor system, and how an executive function such as response inhibition is implemented via connecting circuitry. They suggest that the behavioral requirement to inhibit a response has its counterpart in a neurocognitive inhibitory mechanism that actively suppresses the go command. This bears on the important question of whether "inhibition" is a valid concept in psychological research (see Aron, 2007; MacLeod, Dodd, Sheard, Wilson, \& Bibi, 2003).

An interesting possibility is that this "control circuit," or one that works along similar lines, could be recruited during performance of a wide variety of tasks. Thus, observed activation of such brain regions as the right IFC and STN region can then provide evidence for whether an inhibitory mechanism is operational during planning and switching and other control situations, even when rapid stopping is not required. (It is important to bear in mind, however, that inferring whether a cognitive process is active on the basis of brain activation is limited by the fact that particular brain regions are not activated by one type of cognitive process only; Poldrack, 2006).

\section{THE WIDER FUNCTIONAL NEUROANATOMIC NETWORK}

As we saw, one of the problems that has confounded executivefunction research is the poor correspondence between functional terms such as "inhibition," "maintaining," or "monitoring" and underlying brain regions. By identifying critical nodes such as the right IFC and the STN region with well-defined hypothetical functions (such as inhibition), it is possible to ask what other regions they are connected to, and thus build up a network of putative interacting functional regions. We found that both the right IFC and the STN are connected to the preSMA (part of the superior frontal lobe, adjacent and dorsal to the anterior cingulate) in the right hemisphere (Aron et al., 2007; Fig. 1d). This is striking because preSMA damage also affects stopping (Floden \& Stuss, 2006; Nachev et al., 2007).

While the proper functional role of the preSMA continues to draw considerable research interest, its role could, as a first approximation, be described as "monitoring and resolving response conflict" (e.g., Nachev et al., 2007). This putative function fits nicely with its being a third node in the response-control system, because it helps to solve the riddle of how control is generated. It has often seemed that, to explain how control works, a controller (i.e., a homunuculus/homuncula) needs to be posited. But if there is such a controller, then what controls it? This leads to an infinite regress. Instead, it has been proposed that a monitoring function could constantly check for response conflict-as when multiple possible responses are generated by the current stimulus - and the conflict mechanism could then trigger the controlling processes accordingly (e.g. Botvinick, Cohen, \& Carter, 2004; see also Norman \& Shallice, 1986).

Thus, one possibility is that the preSMA may monitor for conflict between an intended response and a countervailing signal (for an alternative response, or for no response). Then, when such conflict is detected, the "brakes" could be put on via the connection between the right IFC and the STN region. This may explain how responses are completely stopped, and also how they are slowed, pending a decision. When we presented irrelevant stop signals to subjects, we found that their responses on go trials were slowed, without complete cancellation. Moreover, the degree of slowing corresponded to the degree of activation in the right preSMA, the IFC, and the STN region (Aron et al., 2007). Thus, braking (slowing) a response and stopping (canceling) a response may occur via the same system. This is important because many requirements for control, e.g., in Stroop and other interference tasks, are not so much about stopping outright as about, ostensibly, braking an inappropriate response tendency until a discrepancy can be resolved (Frank, 2006). Future experiments could address whether the above-mentioned control circuitry is recruited by, for example, Stroop interference, which would thereby help to clarify its cognitive constituents.

Overall, these results demonstrate how different putative functions can be localized to different brain regions within an overall structurally connected network. The results also provide testable predictions about the functional neuroanatomy underlying other kinds of tasks, such as Stroop interference, planning, and switching.

\section{SWITCHING BETWEEN TASKS OR RESPONSES}

Consistent with the possibility that the revealed circuitry between the right preSMA, IFC, and STN regions has wider applicability, it has been shown that the ability to switch between different tasks or responses also engages key nodes in this network. "Switching" refers to the ability to intentionally change from a task such as answering a phone, to visually searching for something, to doing something else. It has been carefully studied with sophisticated cognitive-psychology paradigms in which subjects perform a series of trials of task A and then switch to performing a series of trials of task B (reviewed by Monsell, 2003). For each subject, engagement of executive functions is measured in terms of the switch cost, which is computed by subtracting the average reaction time of nonswitch trials from the average reaction time of switch trials. While it is still unclear exactly which cognitive processes underpin the switch cost, it 
seems that one constituent process could relate to the control of irrelevant response tendencies, as patients with damage to the right IFC (who had impaired SSRT in the stop-signal paradigm) also had the longest switch costs (reviewed in Aron, 2007; also see Hodgson et al., 2007).

But where does the signal to switch come from? While questions about the origin of control are mysterious, it is intriguing to note that disruption of the preSMA by transcranial magnetic stimulation affected switch trials but not nonswitch trials (Rushworth, Hadland, Paus, \& Sipila, 2002). Further, a recent study recorded directly from neurons in the preSMA of monkeys while they performed an oculomotor switch from a well-practiced (automatic) task to a less practiced one (Isoda \& Hikosaka, 2007b). Switch trials were associated with increased activity of preSMA cells. Moreover, their activity fell within a sufficiently short duration to influence behavior, and also at a time scale that preceded increased switch-related activity within STN neurons in the same paradigm (Isoda \& Hikosaka, 2007a). The authors also showed that injecting electric current into preSMA neurons led to a greater proportion of successful switch trials. In a study of a single patient with selective preSMA damage, an impairment was found in stopping one response and performing another one (Nachev et al., 2007; also see Floden \& Stuss, 2006). Together, these studies strongly suggest that the preSMA is important for switching. Combined with the finding that the right IFC is also important for switching and with the revealed threeway white-matter network between the preSMA, the IFC, and the STN, it could be postulated that the functional mechanism that brakes and stops responses could also underlie switching between tasks. Therefore, this three-way functionalanatomic system for response control does apparently have generality for different effectors (eye, speech, and hand) as well as for different tasks (stopping and switching at least, and maybe others).

\section{CONCLUSIONS}

Innovations in cognitive psychology, embodied in stop-signal and task-switching paradigms, have been keenly adopted by neuroscientists. The combined approach has enabled a finer dissection of executive functions than was possible with classical paradigms such as the WCST. The emerging picture is that performance of control tasks depends on different anatomically connected brain regions, making up a network. As a first approximation, different nodes in the network can be assigned different executive functions. These include "inhibition" for the right IFC and the right STN and "conflict monitoring and resolution of competition" for the preSMA. It is likely that other regions such as the dorsolateral PFC are important for maintaining goals and rules (reviewed by Bunge, 2004) and that other regions are important for maintaining attention, computing the value of outcomes, and adjusting behavior accordingly. Although the picture of how, when, and for which tasks such functions interact is still incomplete, the assignment of such putative functions as inhibition and conflict monitoring/resolution to discrete but connected nodes within the frontal cortex, and the specification of functional connections of these nodes to the subcortex, represents an advance in psychological understanding. As we have seen, these results bear on important controversies such as whether inhibition is a valid construct in psychology, whether responses are controlled by suppressing an agonist or activating an antagonist muscle, what the mechanism underlying Stroop interference is, what the cognitive constituents of the switch cost are, and how exactly the control homunculus can be banished. Future progress will come from further interaction between cognitive psychology and neuroscience. The development of a new generation of cognitive tasks with well-operationalized functional components could do much to complement neuroscience investigations with increasing spatial and temporal resolution.

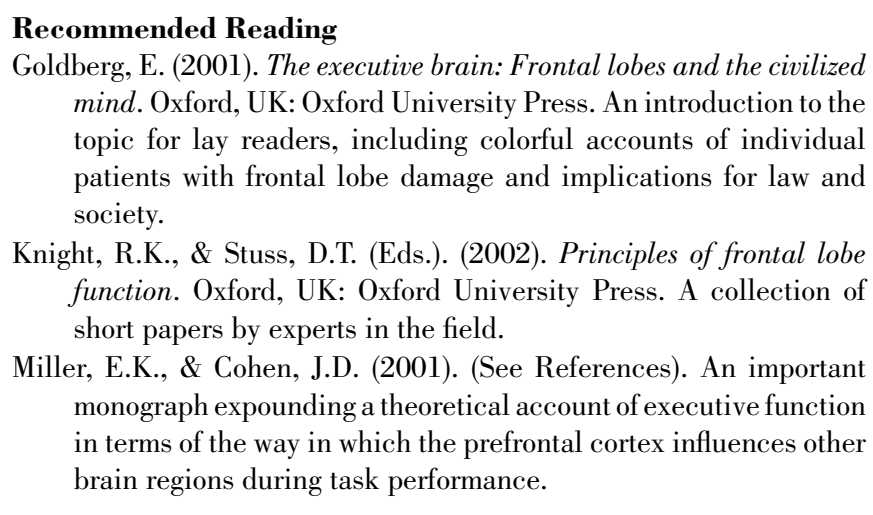

Acknowledgments_-Thanks to David Badre and Vic Ferreira for helpful comments and to Bill Lanouette for editing assistance.

\section{REFERENCES}

Aron, A.R. (2007). The neural basis of inhibition in cognitive control. Neuroscientist, 13, 214-228.

Aron, A.R., Behrens, T.E., Smith, S., Frank, M.J., \& Poldrack, R.A. (2007). Triangulating a cognitive control network using diffusionweighted magnetic resonance imaging (MRI) and functional MRI. Journal of Neuroscience, 27, 3743-3752.

Aron, A.R., Durston, S., Eagle, D.M., Logan, G.D., Stinear, C.M., \& Stuphorn, V. (2007). Converging evidence for a fronto-basal-ganglia network for inhibitory control of action and cognition. Journal of Neuroscience, 27, 11860-11864.

Aron, A.R., \& Poldrack, R.A. (2006). Cortical and subcortical contributions to Stop signal response inhibition: Role of the subthalamic nucleus. Journal of Neuroscience, 26, 2424-2433.

Botvinick, M.M., Cohen, J.D., \& Carter, C.S. (2004). Conflict monitoring and anterior cingulate cortex: An update. Trends in Cognitive Sciences, 8, 539-546. 
Bunge, S.A. (2004). How we use rules to select actions: A review of evidence from cognitive neuroscience. Cognitive, Affective, \& Behavioral Neuroscience, 4, 564-579.

Coxon, J.P., Stinear, C.M., \& Byblow, W.D. (2006). Intracortical inhibition during volitional inhibition of prepared action. Journal of Neurophysiology, 95, 3371-3383.

Floden, D., \& Stuss, D.T. (2006). Inhibitory control is slowed in patients with right superior medial frontal damage. Journal of Cognitive Neuroscience, 18, 1843-1849.

Frank, M. (2006). Hold your horses: A dynamic computational role for the subthalamic nucleus in decision making. Neural Networks, 19 , 1120-1136.

Hodgson, T., Chamberlain, M., Parris, B., James, M., Gutowski, N., Husain, M., et al. (2007). The role of the ventrolateral frontal cortex in inhibitory oculomotor control. Brain, 130, 15251537.

Isoda, M., \& Hikosaka, O. (2007a, November). Role for the subthalamic nucleus in switching from automatic to controlled behavior. Paper presented at the annual meeting of the Society for Neuroscience, San Diego, CA.

Isoda, M., \& Hikosaka, O. (2007b). Switching from automatic to controlled action by monkey medial frontal cortex. Nature Neuroscience, 10, 240-248.

Logan, G.D., \& Cowan, W.B. (1984). On the ability to inhibit thought and action: A theory of an act of control. Psychological Review, 91, $295-327$.
MacLeod, C., Dodd, M., Sheard, E., Wilson, D., \& Bibi, U. (2003). In Opposition to Inhibition. In B.H. Ross (Ed.), The psychology of learning and motivation (Vol. 43, pp. 163-214). San Diego: Elsevier Science.

Miller, E.K., \& Cohen, J.D. (2001). An integrative theory of prefrontal cortex function. Annual Review of Neuroscience, 24, 167-202.

Monsell, S. (2003). Task switching. Trends in Cognitive Sciences, 7, 134140.

Nachev, P., Wydell, H., O’Neill, K., Husain, M., \& Kennard, C. (2007). The role of the pre-supplementary motor area in the control of action. Neuroimage, 36(Suppl. 2), T155-T163.

Norman, D., \& Shallice, T. (1986). Attention of action: Willed and automatic control of behavior. In R.J. Davidson, G.E. Schwartz, \& D. Shapiro (Eds.), Consciousness and self-regulation: Advances in research and theory (Vol. 4, pp. 1-18). New York: Plenum.

Poldrack, R.A. (2006). Can cognitive processes be inferred from neuroimaging data? Trends in Cognitive Sciences, 10, 59-63.

Rabbitt, P.M. (Ed.). (1997). Methodology of frontal and executive function. Hove, UK: Psychology Press.

Robbins, T. (1996). Dissociating executive functions of the prefrontal cortex. Philosophical Transactions of the Royal Society of LondonB: Biological Sciences, 351, 1463-1471.

Rushworth, M.F.S., Hadland, K.A., Paus, T., \& Sipila, P.K. (2002). Role of the human medial frontal cortex in task switching: A combined fMRI and TMS study. Journal of Neurophysiology, 87, $2577-2592$. 